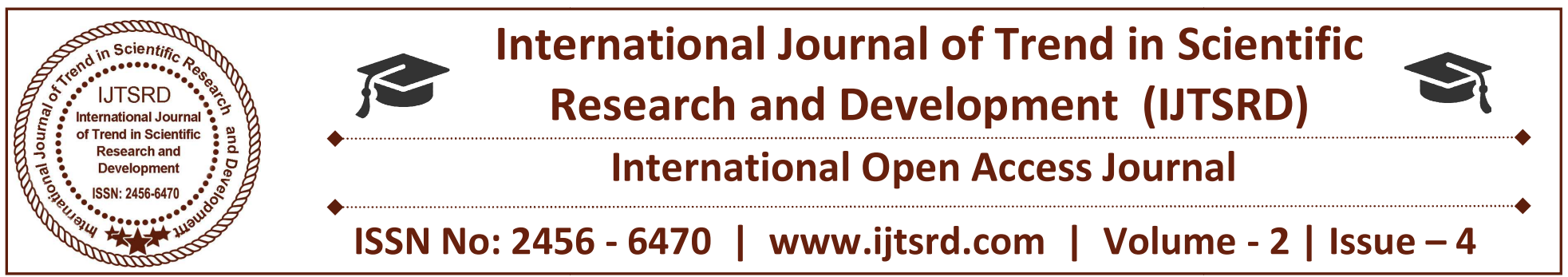

\title{
Evaluation Study of Common Map Users Requirements
}

\author{
Dr. Nagi Zomrawi Mohammed \\ Associate Professor of Surveying and Digital \\ Mapping, Sudan University of Science and \\ Technology, Khartoum, Sudan
}

\author{
Dr. Eiman Eisa Ahmed Elhaj \\ Assistant Professor, Ministry of Physical Planning, \\ Khartoum, Sudan
}

\begin{abstract}
Map producer always try to produce maps that satisfy user needs and requirements. These needs varies from one user to another according to the field of application. Some users concentrate on cartographic requirement while others on geometric one. On the other hand, non-specialist users may not know specific requirement for their application. Knowledge of common users map quality requirements may help map producer to produce maps with general requirement thus, serves large sector of map users community and reduces cost. Moreover, it may reflect the type of map uses in a particular society.

This research work aimed to study the common map quality required by different users in Khartoum. A questionnaire containing different items of map requirements was distributed to a wide spectrum of map users. Results reflects that all users concentrates on only few parameters. They all agreed on map orientation, scalling and only planmetric measurement. While, some parameters such as magnetic declination, overlapping and using standard coulors are not of their interest. On the other hand, about $50 \%$ of map users don't require vertical or topographic information in their field of applications.
\end{abstract}

Keywords: Accuracy, CAAD system, GIS, GPS, Map projection, Map quality, Map requirements

\section{INTRODUCTION}

Maps can be defined as a graphical representation of geospatial data, that is refer to the location or the attributes of object or phenomena on the earth. Maps help their users to better understand geospatial relationship. It give information of distance, direction and area. Size can be retrieved, patterns revealed, and relation understood and quantified. Descriptive information can be also provided on the map.

Features are represented using various combinations of points, lines and symbols. Maps have traditionally been produced in graphic form (hard-copy) that is printed on paper or stable-based plastic material. Today, however, most mapping data is collected in digital form, and is then, processed using Computer Aided Design and Drafting (CADD) systems to develop softcopy maps.

Computer stored softcopy maps can be analyzed, modified, enlarged or reduced in scale in both, soft or hardcopy format. Different types of layers of information can also be extracted from digital maps to be represented and analyzed separately. Moreover, softcopy maps can be instantaneously transferred electronically. Of course, they can also be printed in hardcopy form if desired. Softcopy maps are important in the development and operation of modern Geographic Information Systems ${ }^{[6]}$.

According to field of application, maps can be topographic or planimetric. Topographic maps show natural land features such as lakes, rivers, and mountain peaks as well as man-made features such as roads, railroad tracks, and canals. These maps also contain vertical information represented as contour lines that trace the outline of the terrain and show elevation. Where, planimetric maps don't provide much information about the terrain. Lakes, rivers, and 
mountain pass elevations may be shown, but there isn't any detailed land information ${ }^{[5]}$.

\section{MAP SPECIFICATION REQUIREMENTS}

Map specifications are a set of conditions that the ondemand map should satisfy. In the context of generalization, such conditions have long been represented through cartographic constraints ${ }^{[2]}$.

Expert cartographers are the primary source of this knowledge. They describe the map specifications they judge usable for certain user requirements, but also compliant with the standards of cartographic design.

Maps should satisfy the basic elements and requirements that may include the following:

a. Title that comprises a short description about the purpose of the map. Map title comprises and will includes council name/logo, title, name of map, and map sheet reference which, is a shortened form of the map identification number for each map.

b. Legend which is the principal reference to the map symbols. However, this is still a key element for map reading describing all features.

c. Scale which represent the ratio between map and ground measurements. Sometimes, topographic maps of a scale of 1:40,000 are considered largescale maps, whereas maps of a scale of 1:80,000 or greater are classed as small-scale maps. Largescale maps show more geographic detail than small-scale maps and require more tiles to cover a geographic area.

d. Projection that allows the map producer to represent a portion of the three dimensional curved surface of the earth on a two dimensional paper. Map should include information about map projection used.

e. North Direction that should be oriented - if possible - with north facing up the page. While there is no standard for north point. Magnetic deviation relative to the true north may also be presented on the map.

f. Overlapping or locality map which shows the location of areas covered by an individual sheet relative to the rest of adjacent areas.

g. Grid system that helps the map user to define feature positions.

h. Map Index that must be clear and easily to the map user.

i. Date, to represent the preparation date of individual map sheet ${ }^{[5]}$.
For spatial data accuracy usually, a statistical and testing methodology is implemented for estimating the positional accuracy of points on maps and in digital geospatial data, with respect to georeferenced ground positions of higher accuracy testing methodology and reporting requirements.

Most mapping agencies use the Root Mean Square Error (RMSE) to estimate positional accuracy. RMSE is the square root of the average of the set of squared differences between dataset coordinate values and coordinate values from an independent source of higher accuracy for identical points.

Accuracy reported at the $95 \%$ confidence level means that $95 \%$ of the positions in the dataset will have an error with respect to true ground position that is equal to or smaller than the reported accuracy value. Accuracy test guidelines according to an independent source of higher accuracy is the preferred test for positional accuracy.

Horizontal accuracy shall be tested by comparing the planimetric coordinates of well-defined points in the dataset with coordinates of the same points from an independent source of higher accuracy. Vertical accuracy shall be tested by comparing the elevations in the dataset with elevations of the same points as determined from an independent source of higher accuracy. Errors in recording or processing data, such as reversing signs or inconsistencies between the dataset and independent source of higher accuracy in coordinate reference system definition, must be corrected before computing the accuracy value.

Mapping agencies also have their own requirements and standards for cartographic work including map design and layout.

As a matter of fact, most map design wizards are essentially collecting specifications. This is feasible for simple maps if the background data is predefined and requires limited processing.

\section{MAP PRODUCTION}

The traditional definition of map as a symbolized representation of the ground surface to scale divides map production into two steps; data collection and data presentation.

Data collection varies from direct ground techniques with different accuracy to indirect non-ground techniques such as aerial and satellite imagery, with different resolution. The choice between all these available techniques depends on number of 
parameters including size of the area to be mapped, map scale and accuracy required.

Data presentation may include preservation of appearance and improvement of legibility. Both techniques represent a part of cartographic responsibilities.

Azimuthal projections, conical projections, and cylindrical projections can be used to re-project the earth surface into a two dimensional plane. All these types of projection can be normal to the earth surface, transverse or oblique. Moreover, all these types can be divided into sub-type projection in order to satisfy a particular requirement such as preservation of area; preservation of shape; preservation of scale; preservation of bearing, and ease of drawing ${ }^{[3]}$.

Maps also need to use symbols, colours and letters. It needs some sort of design, generalization of features and other cartographic requirement. Therefore, well cartographic work can leads the better mapping process.

One of the golden rules in mapping process before starting to make field observation is to estimate the accuracy required according to map scale. Then selecting the suitable instruments according to map accuracy specifications.

Figure (1) below is a schematic diagram illustrating general steps of map production.

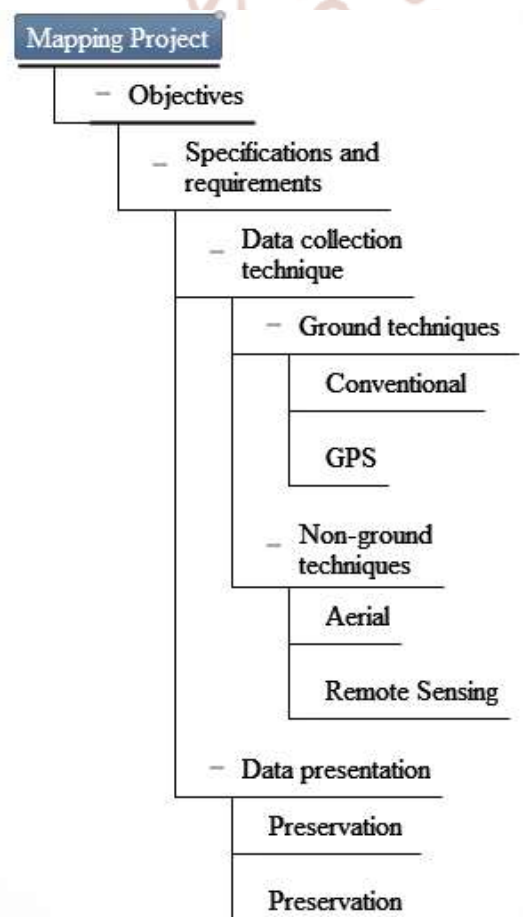

Fig:1 General steps of map production.

\section{TYPES OF MAPS}

Maps can be classified from different points of views such as scale and information contained. In general, there are three broad categories of maps. These are reference, thematic and Special-purpose maps. The reference or topographic maps, generally show several types of spatial data without specific emphasis on one type over another. Reference maps can vary in their complexity and size, but generally include just the various geographic features that give a picture of the area being mapped, e.g. political boundaries, cities, topographic features and/or transportation routes.

Thematic maps, which as their name means that, type of maps has a specific theme or focus. This group of maps is extremely diverse. Thematic maps can vary in topic, complexity, purpose and kind of representation. They generally show characteristics, or attributes, of features that vary spatially. The attribute can vary in a qualitative or nominal way, e.g. categories of land cover; or the attribute can vary in a quantitative way, e.g. amount of precipitation. Thematic maps can represent data with points, lines, areas or volumes. Using visual variables (e.g. hue, lightness, pattern, shape) that fit logically with both the kind of feature being used for the symbols and the way the attribute varies (qualitatively or quantitatively) is key to representing data in thematic maps.

Special-purpose maps are a third category of maps. They lie somewhat between reference maps and thematic maps as they are often reference-like in their use but are made for specific types of users or pertain to a specific type of data. Navigational maps, like those in road atlases or nautical/aeronautic charts, are considered special-purpose maps, as are maps for certain industries or occupations, like soil maps, and municipal utility maps ${ }^{[1]}$.

\section{RESULTS AND ANALYSIS}

Cartographers and map producers try to provide map users with maps that satisfy their needs and requirements. However, on demand map production should satisfy all requirements. In this research work, a study of the type of map users in Khartoum and their common requirements, were analyzed. A questionnaire including eighteen elements of common map requirements was distributed to a wide sector of map community users in Khartoum (Sudan). Each map element appeared in the questionnaire have five level of impotence. 
After collecting the requested data, percentage analysis was carried out for each required criteria and the highest level of importance for each requirement was selected. Result of analysis was found and descendingly sorted as illustrated in table (1) below.

Table: 1 Result of analysis

\begin{tabular}{|c|l|c|}
\hline No. & Map Requirement & Level of Importance \\
\hline \hline 1 & North Arrow & 89 \\
\hline 2 & Accuracy & 78 \\
\hline 3 & Map Title & 67 \\
\hline 4 & Scale & 67 \\
\hline 5 & legend & 67 \\
\hline 6 & Reliability & 67 \\
\hline 7 & Source of data & 67 \\
\hline 8 & Lettering & 56 \\
\hline 9 & Georeference & 56 \\
\hline 10 & Elevation & 45 \\
\hline 11 & Conversion of elevation & 45 \\
\hline 12 & Map Index & 45 \\
\hline 13 & Grid & 34 \\
\hline 14 & Glossary & 34 \\
\hline 15 & sheet Number & 33 \\
\hline 16 & Overlapping & 33 \\
\hline 17 & Standard colours & 22 \\
\hline 18 & Magnetic declination & 22 \\
\hline
\end{tabular}

From results obtained in the above table, it can obviously seen that most of map user's society concentrates on the existence of the north direction arrow that is important for correct map orientation.

Map requirements that concerned with taking planimetric measurements from the map such as accuracy, source of data, reliability and map scale, are also important for most of map users. Where, topographic information are not required by most map users.

For map reading and extracting descriptive information, users concentrates only on the title, legend and lettering.

In reverse, some of the map society users underestimate importance of existence of the map title and the north direction in the map which is strange.

Results also reflects that, using standard colours, magnetic declination, sheet number, overlapping, glossary and grid are not so important for most users. Therefore, such map requirements should not given priority by map producer and can be included in the map on demand.
Producing maps that satisfies general requirements to serve wide sector of map users my helps both map producer and map users in providing maps that's serves large sector of map user with lower cost.

It can be said that, about fifty percent of the studied map requirements were demanded by all map users all of them, concentrates on map requirements that concerned with taking planmetric measurement and extracting descriptive information.

Map users those don't consider sheet numbers and overlapping, used to use single map and have no culture of using series of maps.

Figure (2) below illustrates a bar graph that reflects importance of map requirements that demanded by studied sector of map users.

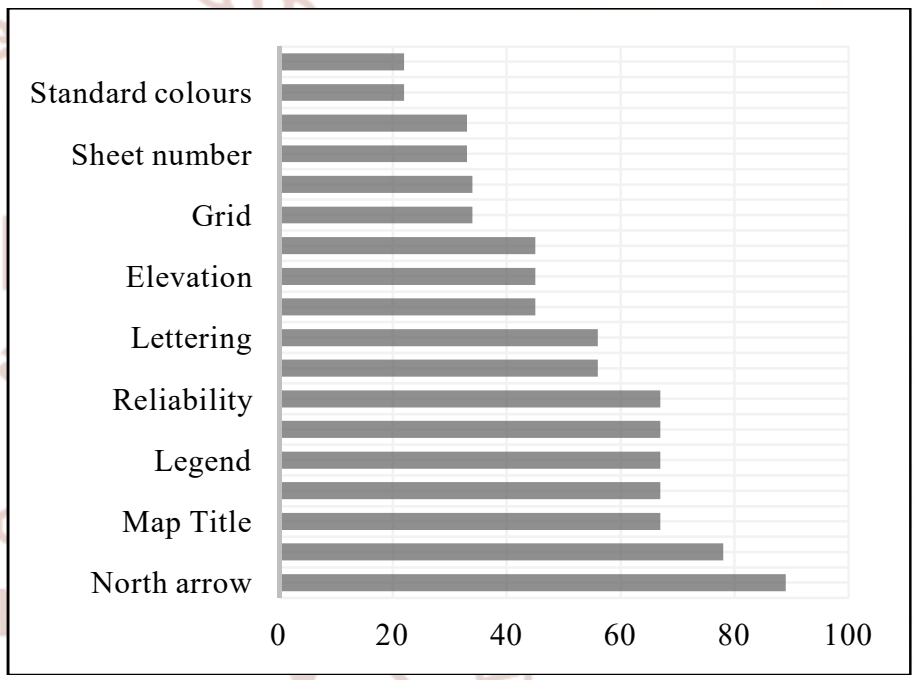

Fig: 2 Graphical presentation of resultant analysis

\section{CONCLUSION}

Not all map specifications and requirements are demanded by map users. Some of these requirements did not sever specific applications. In this study work, data about different users map requirements was collected and analyzed. Results of this study work can be summarized in the following conclusions:

- About fifty percent of the studied map requirements were demanded by all map users.

- Many map users concentrates on map requirements that concerned with taking planmetric measurement and extracting descriptive information.

- Topographic information are not required by most of map users.

- Map requirements such as map grid, magnetic declination, standard colours, and glossary were 
not considered by many map users. Therefore, some of such map requirements could be given no priority by map producer and can be included in the map on demand.

- Many map users do not have the culture of using map series.

- Producing maps that satisfies general requirements may serve map producer in presenting maps that serves wide sector of map user with economical cost.

\section{References}

1) https://www.e-education.psu.edu/geog486/11.html

2) https://www.researchgate.net/publication/2336240 76 WeightSetting_and_Quality_Assessment_in_Simultaneou s_Graphic_Generalization.
3) Nagi Zomrawi (2015), Surveying the state-of-the art, International journal of engineering sciences \& management. October December.

4) Nagi Zomrawi (2017), Nagi methods of map quality evaluation, International Journal of Engineering and Advanced Technology Studies, November.

5) Nagi Zomrawi, (2013), A New Approach of Map Quality Evaluation Applied to Khartoum State Survey Authority (Sudan), International journal of multidisciplinary sciences and engineering, Vol.4 May. 7

6) Rbinson, and others (1995), Element of Cartography, New York, John Wily and Sons.

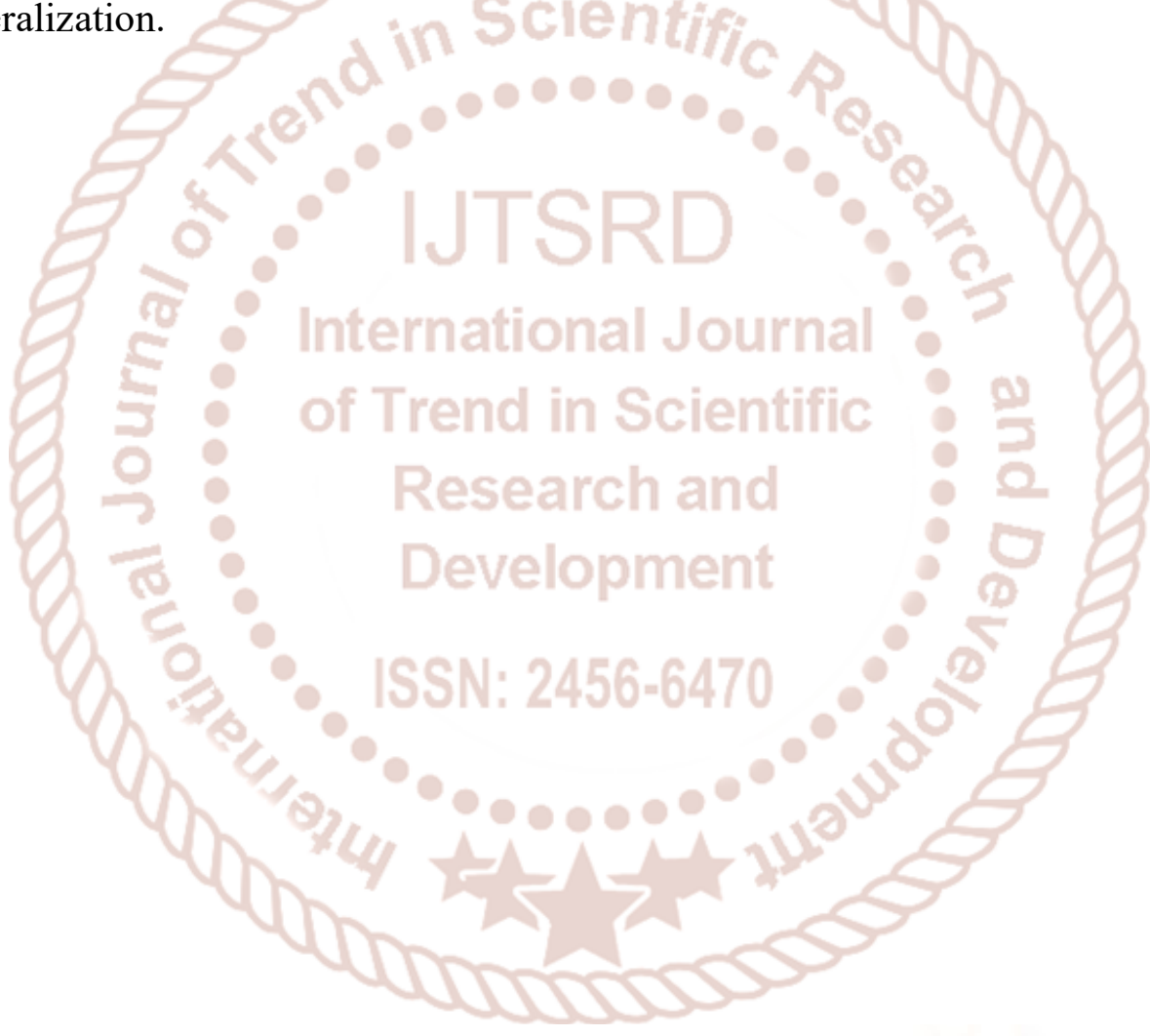

